\title{
Recent Advances in Small Animal SPECT Instrumentations and Techniques
}

\author{
Francesco Cusanno*, Michele Ballerini, Evaristo Cisbani, Stefano Colilli, Rolando \\ Fratoni, Franco Garibaldi, Fausto Giuliani, Massimo Gricia, Maurizio Lucentini, \\ Fabio Santavenere, Sergio Torrioli and Pietro Veneroni \\ Istituto Superiore di Sanità, Rome, Italy \\ E-mail: francesco.cusannodiss.infn.it, \\ michele.ballerinieiss.infn.it, evaristo.cisbani@iss.infn.it, \\ stefano.colillieiss.infn.it, franco.garibaldi@iss.infn.it, \\ fausto.giulianidiss.infn.it, fabio.santavenerediss.infn.it \\ sergio.torriolidiss.infn.it
}

\section{Maria Lucia Magliozzi}

Istituto Nazionale di Fisica Nucleare and Univerità La Sapienza, Rome, Italy

E-mail: lucia.magliozzi@iss.infn.it

\section{Stan Majewski}

Jefferson Lab, Newport News VA, USA

E-mail: majewski@jlab.org

\section{Seng Peng Greta Mok, Benjamin M. W. Tsui and Yuchuan Wang}

Johns Hopkins University, Baltimore MD, USA

E-mail: smok@jhsph. edu, btsui1@jhmi .edu, yuchuan@jhmi . edu

\begin{abstract}
This paper reports on the most advanced evolutions of SPECT detectors for molecular imaging, with the specific goal of the visualization of atherosclerotic plaques in mice. An extensive Monte Carlo simulation has been performed to choose the best detector configuration to match the requirements in terms of spatial resolution and sensitivity for this aim. Simulations, prototype tests and prelimary measurements with transgenic mice are reported.
\end{abstract}

Frontiers in Imaging Science: High Performance Nuclear Medicine Imagers for Vascular Disease Imaging (Brain and Heart)

November 13-14 2006

Istituto Superiore di Sanità, Rome, Italy

\footnotetext{
* Speaker.
} 


\section{Introduction}

The role of SPECT technique in molecular imaging is unique, since it can provided both submillimeter spatial resolution, picomolar sensitivity, and functional information. Compared to PET technique, SPECT does not have intrinsic limitation for spatial resolution and usually allows longer studies due to the longer life-time of the radionuclides, but it has much less sensitivity due to the passive collimation. Therefore design of new collimation techniques as multi-pinholes or coded apertures play a key role in SPECT [1,2].

Small animals such as rats and mice may be used to model human diseases and allow studies of human pathologies under controlled conditions. Mice are particularly useful because most of human genes have a related mouse gene and many transgenic mice are currently available [3]. Successful biomedical studies of the myocardium and of skeletal bone in mice have been conducted with pinhole SPECT obtaining excellent spatial resolution and affordable counting rate $[4,5]$.

\section{Application: Atherosclerotic Plaques}

Atherosclerotic plaques, a disease causing most of deaths in western countries, are accumulation of connective tissue, lipids, cholesterol, macrophages on the arterial walls. They develop slowly, silently and very often asymptomatically.

The vulnerable plaques have high probability of rupture and therefore produce suddendly the atherosclerosis as ischemic stroke or infarction.

This kind of plaques has peculiar characteristics in term of composition and metabolism. Traditional diagnostic techniques (as ultra-sound Doppler and angiography) are not specific and do not provide information on plaque composition or metabolism, thus they can not help the identification of the vulnerable ones.

Hypothesis has been formulated on the presence of significant apoptosis in vulnerable plaques. Annexin-V protein has a noticeable uptake from apoptotic cells and therefore ${ }^{99 m} T c$-labelled Annexin$\mathrm{V}$ can be exploited by SPECT to verify this hypothesis and, if the hypothesis is true, to visualize vulnerable plaque.

ApoE -/- transgenic mice feeded with fatty diet develop atherosclerotic plaques and can be used as model for the aim. Maximum plaque size in the mouse aorta is about $0.5 \times 1 \times 4 \mathrm{~mm}^{3}$ and the expected ${ }^{99 m} T c$ uptake is around $10 \mathrm{kBq}$ : sub-millimeter spatial resolution keeping adequate sensitivity are required for "in-vivo" studies.

\section{Monte Carlo Simulations}

For obtaining the best spatial resolution keeping high efficiency in a SPECT system, different design for scintillators and photodetectors are possible. Light yield, light distribution on photodetector, light wavelength, and light sampling can be critical to obtain the best results. In order to design the best gamma detector for SPECT system, an extensive Monte Carlo simulation using GEANT 4 package [6] has been performed [7]. Continuous $\mathrm{LaBr}_{3}: \mathrm{Ce}$ and segmented NaI:Tl, CsI:Tl and CsI:Na scintillators has been considered with a light sampler ranging from $1.5 \mathrm{~mm}$ to $6 \mathrm{~mm}$. According to the manufacturing limit, we considered NaI:Tl with pixel ranging from $1.0 \mathrm{~mm}$ 

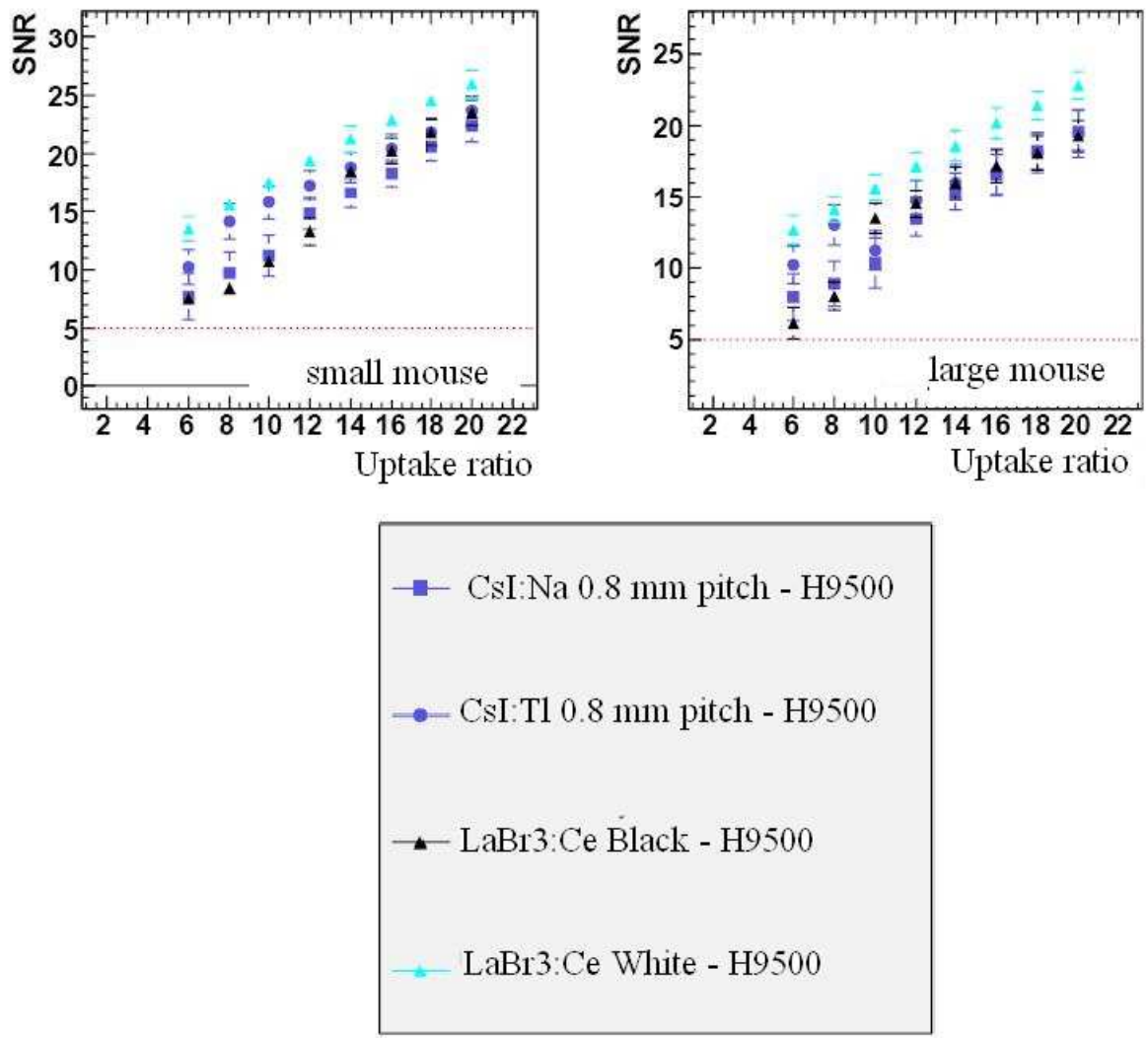

Figure 1: Signal-to-Noise Ratio SNR for $0.5 \times 1 \times 4 \mathrm{~mm}^{3}$ atherosclerotic plaque in mouse versus uptake ratio. $0.3 \mathrm{~mm}$-diameter pinhole $(\mathrm{PH})$ and magnification $(\mathrm{M})$ of 3 are considered. For $\mathrm{LaBr}_{3}$ scintillator, absorbing (Black) and diffusive (White) entrance windows are simulated. According to the manufacturers, $\mathrm{LaBr}_{3}$ is considered directly coupled to photodetectors (integral line), CsI:Na is considered encapsulated in $3-m m$ thick glass, CsI:Tl is considered directly coupled to photodetectors. Two different background volumes are considered to take into account variations of the mouse size. The plot on the left refers to $15 \mathrm{~mm}$ diameter mouse ("small mouse"), the plot on the right refers to $40 \mathrm{~mm}$ diameter mouse ("large mouse").

to $1.5 \mathrm{~mm}$ and CsI:Tl and CsI:Na with pixel ranging from $0.4 \mathrm{~mm}$ to $1.0 \mathrm{~mm}$, with $0.8 \mathrm{~mm}$ gap between adjacent pixels. Results of the simulations shows that $\mathrm{LaBr}_{3}: \mathrm{Ce}$ continuous crystal will provide better spatial resolution keeping good efficiency. Pixellated $\mathrm{NaI}: \mathrm{Tl}$ can not reach $\mathrm{LaBr}_{3}: \mathrm{Ce}$ spatial resolution, while CsI:Tl and CsI:Na can not keep the same efficiency with so small pixel size to reach spatial resolution as good as with $\mathrm{LaBr}_{3}: \mathrm{Ce}$. Furthermore, $\mathrm{LaBr}_{3}: \mathrm{Ce}$ has much higher pulse heigh and hence much better energy resolution. In addition, a continuous scintillator does not require very small light sampling as for very small pixel of segmented crystals. Better performance provide Better Signal-to-Noise Ratio in imaging atherosclerotic plaques in mice. Fig. 1 shows the Signal-to-Noise ratio as function of the uptake ratio for $0.5 \times 1 \times 4 \mathrm{~mm}^{3}$ atherosclerotic plaque in a mouse using $100 \times 100 \times 3 \mathrm{~mm}^{3}$ scintillator modules coupled to $2 \times 2$ array of HPK H9500 Flat Panels. $600 \mathrm{~Bq} / \mathrm{mm}^{3}$ uptake of ${ }^{99 m} \mathrm{Tc}$ is assumed as background from tissue surrounding the plaque. 


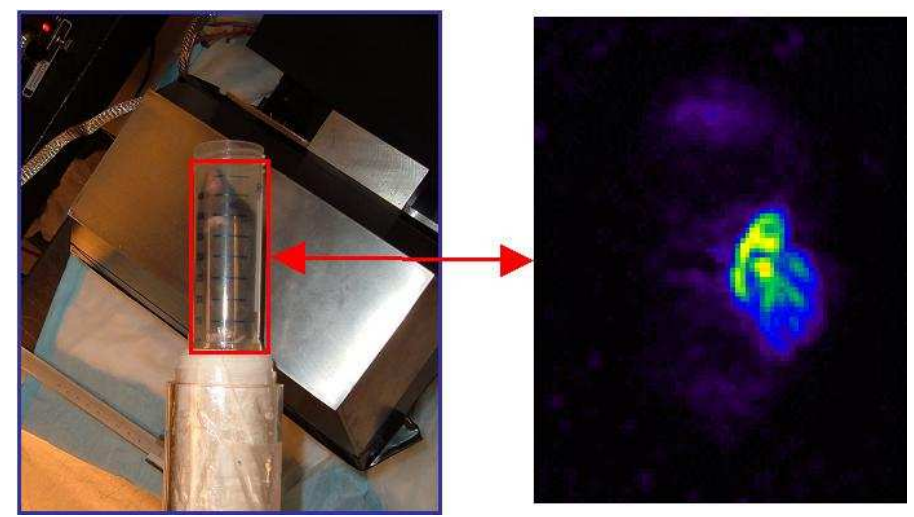

Figure 2: Image of mouse boby after injection of ${ }^{99 m} T c$-Annexin V. Atherosclerotic plaques are not visible, very probably due to the youngness of the mouse (see text). Uptake of Annexin V on the kidneys is evident, showing the excellent spatial resolution and image quality.

\section{Preliminary Studies at Johns Hopkins University}

Explorative measurement on young ApoE -/- mouse has been conducted at Johns Hopkins University, small animal facility, with a prototype system based on single tungsten pinhole $0.3 \mathrm{~mm}$ diameter. We tested both NaI:Tl $1.2 \mathrm{~mm}$ pixel and CsI:Tl $1.0 \mathrm{~mm}$ pixel scintillators coupled to a $2 \times 2$ array of HPK H9500 Flat Panels. Magnification factor of 3 was used, corresponding to single-view FOV of $33 \times 33 \mathrm{~mm}^{2}$. The used readout is based on 5053 IDEAS cards with their VATAC data-acquisition system [8]. Using the CsI:Tl scintillator, with measured total spatial resolution of $0.6 \mathrm{~mm}$, we imaged a 15 -week-old mouse after injection of $70 \mathrm{MBq}$ of ${ }^{99 m} \mathrm{Tc}$-Annexin V. The mouse was keeped anesthetized with continuous gas flowing. 64 one-minute views were performed. Maximum-Intensity-re-Projection alghoritm was used to reconstruct the 3D image. Usually ApoE -/- mice develop vulnerable atherosclerotic plaques starting from the 18-th week, therefore very probably the mouse was too young to show plaques. Nevertheless, very good image was obtained due to the strong Annexin-V uptake in the kidneys (Fig. 2), showing the potential of the technique.

\section{Conclusions}

Molecular imaging with radionuclides is a very powerful technique allowing studies of "invivo" biological processes. Single-photon modality allows longer studies than PET and could use different tracers, with no intrinsic limit in spatial resolution. Advanced SPECT detectors are required in order to obtain sub-millimeter spatial resolution keeping adequate sensitivity for example in the study of atherosclerotic plaques in mice.

\section{References}

[1] R. Accorsi et al., A coded aperture for high-resolution nuclear medicine planar imaging with a conventional anger camera: experimental results, IEEE Trans Nucl Sci 48 (6) (2001) 2411-2417. 
[2] F. Beekman et al., Design and simulation of a high-resolution stationary SPECT system for small animals, Phys Med Biol 49 (2004) 4579-4592.

[3] T. F. Budinger et al., Imaging transgenic animals, Annu Rev Biomed Eng 01 (1999) 611-648.

[4] K. Ishizu et al., Ultra-high resolution SPECT system using four pinhole collimators for small animal studies, J Nucl Med 36 (1995) 2282-87.

[5] S. E. Strand et al., Small animal imaging with pinhole single-photon emission computed tomography, Cancer 73 (1994) 981-84.

[6] S. Agostinelli et al., Geant4 - a simulation toolkit, Nucl Instr Meth A506 (2003) 250-303.

[7] E. Cisbani et al., Molecular imaging with radionuclides, a powerful technique for studying biological processes in vivo, Nucl Instr Meth A571 (2007) 169-172.

[8] F. Cusanno et al., Molecular breast imaging: First results from Italian- National-Institute-of-Health clinical trials, Nucl Instr Meth A571 (2007) 77-80. 